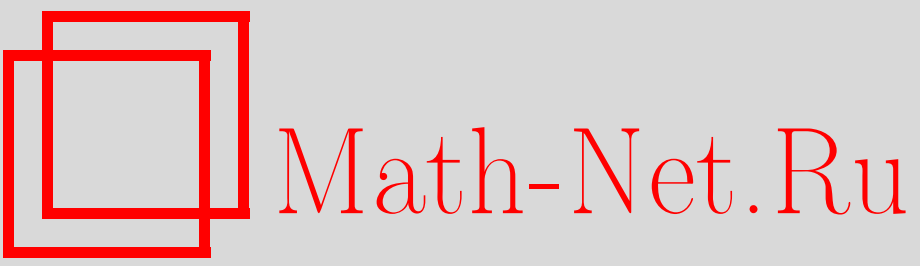

P. Bertail, S. Clémencon, Sharp bounds for the tails of functionals of Markov chains, Теория вероятн. и ее примен., 2009, том 54, выпуск 3, 609-619

DOI: https://doi.org/10.4213/tvp2815

Использование Общероссийского математического портала Math-Net.Ru подразумевает, что вы прочитали и согласны с пользовательским соглашением

http://www . mathnet.ru/rus/agreement

Параметры загрузки:

IP : 54.92 .164 .108

26 апреля 2023 г., 16:10:19 
(C) 2009 г.

BERTAIL P.* , CLÉMENÇON S.**

\title{
SHARP BOUNDS FOR THE TAILS OF FUNCTIONALS OF MARKOV CHAINS
}

\begin{abstract}
В статье устанавливаются точные оценки вероятностей уклонений частичных сумм $\sum_{i=1}^{n} f\left(X_{i}\right)$, где $X=\left(X_{n}\right)_{n \in \mathbf{N}}$ - положительно возвратная цепь Маркова, а $f$ - вещественнозначная функция, определенная на ее пространстве состояний. Комбинируя метод восстановления и преобразование Эшера, мы, в частности, показываем, что полученные нами оценки обобщают вероятностные неравенства, известные ранее для случая независимых одинаково распределенных величин, на марковскую постановку для (не обязательно равномерно) геометрически эргодических цепей.
\end{abstract}

Ключевые слова и фразы: неравенства для вероятностей, цепь Маркова, метод восстановления.

1. Introduction. Consider a positive recurrent Markov chain $X=\left(X_{n}\right)_{n \in \mathbf{N}}$ with transition probability $\Pi$, state space $E$ with countably generated $\sigma$-field $\mathscr{E}$ and stationary distribution $\mu$. It is well known that we may then restrict ourselves to the case when the chain $X$ is regenerative, namely when there exists a measurable set $A$ such that $\mu(A)>0$ and $\Pi(x, \cdot)=\Pi(y, \cdot)$ for all $(x, y) \in A^{2}$, even if it entails considering a Nummelin extension of the initial chain (see [18]). By virtue of the strong Markov property, the sequence $\left(\tau_{A}(n)\right)_{n \in \mathbf{N}}$ of successive passage times to the regeneration set $A$ forms a (possibly delayed) renewal process in this case and the segments $\left(X_{1+\tau_{A}(j)}, \ldots, X_{\tau_{A}(j+1)}\right), j \geqslant 1$, obtained by dividing the sample path of the chain according to the regeneration times, are i.i.d. r.v.'s, valued in the torus $\mathbf{T}=\bigcup_{n \geqslant 1} E^{n}$. Furthermore, the return time to the atom $A$ has finite expectation, and the stationary distribution may be viewed as a Pitman occupation measure:

$$
\forall B \in \mathscr{E}, \quad \mu(B)=\frac{1}{\mathbf{E}_{A}\left[\tau_{A}\right]} \mathbf{E}_{A}\left[\sum_{i=1}^{\tau_{A}} \mathbf{I}_{\left\{X_{i} \in B\right\}}\right],
$$

where $\tau_{A}=\inf \left\{n \geqslant 1: X_{n} \in A\right\}, \mathbf{E}_{A}[\cdot]$ denotes the expectation conditioned on $X_{0} \in A$ and $\mathbf{I}_{\{\mathscr{A}\}}$ the indicator function of any event $\mathscr{A}$. One may refer to [19] for an account on renewal theory applied to Markov chain analysis. Let $f:(E, \mathscr{E}) \rightarrow \mathbf{R}$ be a measurable function. Asymptotic expansions for the tail probabilities of the partial sums $n^{-1 / 2} \sum_{i=1}^{n} f\left(X_{i}\right)$ have been obtained via the regenerative method (see [23]), whose technique consists, roughly speaking, in applying appropriate results for i.i.d. r.v.'s to the partial sums over regeneration cycles $\sum_{i=1+\tau_{A}(j)}^{\tau_{A}(j+1)} f\left(X_{i}\right), j \geqslant 1$. Refer, in particular, to [4], [13], and [3] for such refinements of the central limit theorem. See also the limit theorems for large deviations proved in [16], based on the characteristic function method. This paper aims at establishing, with the same means, nonasymptotic bounds for the probability that the sum $\sum_{i=1}^{n} f\left(X_{i}\right)$ exceeds a prescribed number $x$, holding for all $x$ and $n$, similar to tail bounds proved in the independent framework such as those obtained by W. Hoeffding, G. Bennett or S. V. Nagaev for instance (see [10], [1], and [17], respectively). We point out that the regenerative method is by no means the sole technique for obtaining probability inequalities in the Markovian setting. Such nonasymptotic results may be established by martingale arguments (see [9]), coupling techniques based on decay rate assumptions for

* MODALX-Université Paris X, Nanterre \& CREST (INSEE); e-mail: bertail@ensae.fr

** LTCI UMR GET/CNRS 5141, Paris; e-mail: stephan.clemencon@telecomparistech.fr 
mixing coefficients (see, e.g., [5] or [21]), from results of the quasi-compact operator theory when the spectral gap property is assumed to hold for the transition kernel (see [12] and [11]) or by methods based on information inequalities as in [14] (see also [22]). However, the regenerative method imposes much less restrictions on the ergodicity properties of the chain than most alternative techniques. In particular, it may be used without stipulating Doeblin's condition (i.e., uniform ergodicity) to be fulfilled.

The paper is organized as follows. Section 2 first gives an insight into how the regenerative approach may apply for deriving tail bounds in the positive recurrent case, appealing additionally to an exponential change of probability measure as in [24] and then states the main results of the paper. Technical proofs are postponed to Section 3.

2. Probability inequalities for regenerative Markov chains. We first introduce the notation. Let $\nu$ be some probability distribution on $(E, \mathscr{E})$. Here and throughout, we denote by $\mathbf{P}_{\nu}$ (respectively, $\mathbf{P}_{A}$ ) the probability measure on the underlying space such that $X_{0} \sim \nu$ (respectively, conditioned on $X_{0} \in A$ ) and by $\mathbf{E}_{\nu}[\cdot]$ (respectively, by $\mathbf{E}_{A}[\cdot]$ ) the $\mathbf{P}_{\nu}$-expectation (respectively, the $\mathbf{P}_{A}$-expectation). Furthermore, it is assumed throughout the paper that $\mathbf{E}_{A}\left[\tau_{A}^{2}\right]<\infty$ or equivalently that $\mathbf{E}_{\mu}\left[\tau_{A}\right]<\infty$, since by a standard result in renewal theory $\mathbf{P}_{\mu}\left(\tau_{A}=k\right)=\mu(A) \mathbf{P}_{A}\left(\tau_{A} \geqslant k\right)$ for all $k \geqslant 1$.

For i.i.d. r.v.'s $Y_{1}, \ldots, Y_{n}$ such that $\mathbf{E}\left[Y_{1}\right]=0, \mathbf{E}\left[Y_{1}^{2}\right]=\sigma^{2}$ and $\left|Y_{1}\right| \leqslant M$ for some constant $M$, the following tail estimate was obtained in [1]:

$$
\mathbf{P}\left(\sum_{i=1}^{n} Y_{i} \geqslant x\right) \leqslant \exp \left\{-\frac{n \sigma^{2}}{M^{2}} H\left(\frac{M x}{n \sigma^{2}}\right)\right\},
$$

with $H(x)=(1+x) \ln (1+x)-x$.

When the $Y_{i}$ 's are not bounded anymore, this inequality may be extended using truncation arguments, as in [8] (see also [17]) at the price of an additional term related to the tail behavior of the $Y_{i}$ 's on the right-hand side of (1). For arbitrary positive constants $x$ and $M$, it is established in [8] (see Theorem 4 therein) that

$$
\begin{aligned}
\mathbf{P}\left(\sum_{i=1}^{n} Y_{i} \geqslant x\right) & \leqslant \exp \left\{\frac{x}{M}-\left(\frac{x-n m_{M}}{M}+\frac{n \sigma_{M}^{2}}{M^{2}}\right) \ln \left(1+\frac{x M}{n \sigma_{M}^{2}}\right)\right\}+n \mathbf{P}\left(Y_{1}>M\right) \\
& \leqslant \exp \left\{-\frac{n \sigma_{M}^{2}}{M^{2}} H\left(\frac{M x}{n \sigma_{M}^{2}}\right)\right\}+n \mathbf{P}(Y>M)
\end{aligned}
$$

with $m_{M}=\mathbf{E}\left[Y_{1} \mathbf{I}_{\left\{\left|Y_{1}\right| \leqslant M\right\}}\right]$ and $\sigma_{M}^{2}=\mathbf{E}\left[Y_{1}^{2} \mathbf{I}_{\left\{\left|Y_{1}\right| \leqslant M\right\}}\right]$.

This paper is devoted to establishing analogous inequalities under the assumption that $Y_{i}=f\left(X_{i}\right), 1 \leqslant i \leqslant n$, where $f:(E, \mathscr{E}) \rightarrow \mathbf{R}$ is a $\mu$-integrable function. Without loss of generality, we assume that $\mu(f)=\mathbf{E}_{\mu}\left[f\left(X_{1}\right)\right]=0$ throughout the article.

2.1. The regenerative approach. Let $l_{n}=\sum_{i=1}^{n} \mathbf{I}_{\left\{X_{i} \in A\right\}}$ be the number of renewals (i.e., visits to the regeneration set $A$ ) up to time $n$. We denote by $\alpha=\mathbf{E}_{A}\left[\tau_{A}\right]$ the mean inter-renewal time. For $j \geqslant 1$, define $S_{j}(f)=\sum_{i=1+\tau_{A}(j)}^{\tau_{A}(j+1)} f\left(X_{i}\right)$ and $s_{j}=$ $\tau_{A}(j+1)-\tau_{A}(j)$. Notice that these are two sequences of i.i.d. r.v.'s with common variance $\sigma_{f}^{2}$ and $\sigma_{1}^{2}$, respectively, which are assumed to be finite throughout the article. The regenerative method is based on the preliminary observation that the sum $\sum_{i \leqslant n} f\left(X_{i}\right)$ can be decomposed as follows on the event $\left\{l_{n} \geqslant 2\right\}$ :

$$
\sum_{i=1}^{n} f\left(X_{i}\right)=S_{A}(f)+\sum_{j=1}^{l_{n}-1} S_{j}(f)+S_{n}^{(n)}(f),
$$

where $S_{A}(f)=\sum_{i=1}^{\tau_{A}} f\left(X_{i}\right)$ and $S_{n}^{(n)}(f)=\sum_{i=1+\tau_{A}\left(l_{n}\right)}^{n} f\left(X_{i}\right)$ with the usual convention regarding empty summation. It is noteworthy that the summands $S_{A}(f), S_{1}(f), \ldots, S_{l_{n-1}}(f)$ and $S_{n}^{(n)}(f)$ on the right-hand side of (3) are generally not 
independent for fixed $n$ (observe that, when $f \equiv 1$ for instance, they sum up to $n$ ). Hence, when applied for establishing tail bounds, the regenerative method comprises three main steps. The first one consists in partitioning the underlying space $\Omega$ according to all possible fashions for the chain to regenerate up to time $n$, so that the summands in (3) be mutually independent on each subset of the partition. The matter is next to establish a tail bound on each of these subsets by relying on the resulting independence structure. And the desired tail estimate is finally computed by summing all the bounds previously obtained in a way that the sum obtained can be identified.

Now that an insight into the principle of the method has been given, in the next subsection we turn to developing the argument for proving tail inequalities in a rigorous fashion. Observe first that for all strictly positive real numbers $p_{1}, p_{2}$, and $p_{3}$ such that $1 / p_{1}+1 / p_{2}+1 / p_{3}=1$, we have for all $x>0$ the immediate bound

$$
\begin{aligned}
\mathbf{P}_{\nu}\left(\sum_{i=1}^{n} f\left(X_{i}\right) \geqslant x\right) \leqslant & \mathbf{P}_{\nu}\left(\sum_{i=1}^{n} f\left(X_{i}\right) \geqslant x, l_{n} \leqslant 1\right)+\mathbf{P}_{\nu}\left(S_{A}(f) \geqslant \frac{x}{p_{1}}\right) \\
& +\mathbf{P}_{\nu}\left(\sum_{j=1}^{l_{n}-1} S_{j}(f) \geqslant \frac{x}{p_{2}}\right)+\mathbf{P}_{\nu}\left(S_{n}^{(n)}(f) \geqslant \frac{x}{p_{3}}\right)
\end{aligned}
$$

with the convention that $\sum_{j=1}^{l_{n}-1} S_{j}(f)=0$ when $l_{n} \leqslant 1$. Whereas bounds for the first two terms on the right-hand side of (4), as well as for the last one, can easily be derived from assumptions on the tails of $\tau_{A}$ and $S_{A}(f)$ under $\mathbf{P}_{\nu}$ and $\mathbf{P}_{A}$, special attention must be paid to the tail of $\sum_{j=1}^{l_{n}-1} S_{j}(f)$. Beyond the dependence structure among the summands emphasized above, it is noteworthy that the $S_{j}(f)$ 's are generally unbounded (even though one assumes $f$ to be bounded).

2.2. Main result. Although the technique we present here for establishing bounds for the tail behavior of $\sum_{j=1}^{l_{n}-1} S_{j}(f)$ is very general, we focus on a simple result for the sake of clarity. Precisely, we derive an inequality extending (2), whose right-hand side consists of two components: the first one being of the form of an exponential bound involving truncated moments of the $\left(S_{j}(f), s_{j}\right)$ 's, while the second one is related to their tail behavior. Other probability bounds can be deduced by a slight adaption of the argument described below. And extensions of moment inequalities of Rosenthal type for $\sum_{i \leqslant n} f\left(X_{i}\right)$ can be established with the same method. We also emphasize that, although the present study is confined to the Markovian setting, the method applies in the same way to any (eventually continuous time and not necessarily Markovian) stochastic process with a regenerative extension (see [25]).

Theorem 1. Consider a regenerative positive recurrent Markov chain $X=\left(X_{n}\right)_{n \in \mathbf{N}}$ with initial and stationary probability distributions $\nu$ and $\mu$. Let $A$ be an accessible atom for $X$ and let $f:(E, \mathscr{E}) \rightarrow \mathbf{R}$ be some $\mu$-integrable function. Assume further that

(i) $\alpha=\mathbf{E}_{A}\left[\tau_{A}\right]<\infty$ and $0<\sigma_{A}^{2}=\mathbf{E}_{A}\left[\tau_{A}^{2}\right]<\infty$,

(ii) $\mathbf{E}_{A}\left[S_{A}(f)\right]=0$ and $0<\sigma_{f}^{2}=\mathbf{E}_{A}\left[S_{A}(f)^{2}\right]<\infty$.

Then, for any vector $M=\left(M_{1}, M_{2}\right) \in(\mathbf{R} \backslash\{0\})^{2}$ of Euclidean norm $\|M\|=\left(M_{1}^{2}+\right.$ $\left.M_{2}^{2}\right)^{1 / 2}$, we have, for any $n \geqslant \alpha$,

$$
\begin{gathered}
\mathbf{P}_{\nu}\left(\sum_{j=1}^{l_{n}-1} S_{j}(f) \geqslant x\right) \leqslant C_{M} \exp \left\{-\frac{n(1+|\widetilde{\rho}|) \widetilde{\sigma}^{2}}{2\|M\|^{2}} H\left(\frac{\|M\| \sqrt{2}}{(1+|\widetilde{\rho}|) \widetilde{\sigma} \widetilde{\sigma}_{f}} x\right)\right\} \\
+(n-1) \mathbf{P}_{A}\left(\left|S_{A}(f)\right| \geqslant M_{1}\right)+(n-1) \mathbf{P}_{A}\left(\tau_{A} \geqslant M_{2}\right),
\end{gathered}
$$

with

$$
\begin{aligned}
\tilde{\sigma}_{f}^{2} & =\mathbf{D}_{A}\left(S_{A}(f) \mathbf{I}_{\left\{\left|S_{A}(f)\right| \leqslant M_{1}\right\}}\right), \quad \widetilde{\sigma}_{A}^{2}=\mathbf{D}_{A}\left(\tau_{A} \mathbf{I}_{\left\{\tau_{A} \leqslant M_{2}\right\}}\right), \\
\widetilde{\rho} & =\left(\widetilde{\sigma}_{f} \widetilde{\sigma}_{A}\right)^{-1} \operatorname{cov}_{A}\left(S_{A}(f) \mathbf{I}_{\left\{\left|S_{A}(f)\right| \leqslant M_{1}\right\}}, \tau_{A} \mathbf{I}_{\left\{\tau_{A} \leqslant M_{2}\right\}}\right), \\
\widetilde{\sigma} & =\sqrt{\widetilde{\sigma}_{f}^{2} \widetilde{\sigma}_{A}^{2}\left(\widetilde{\sigma}_{f}^{2}+\widetilde{\sigma}_{A}^{2}\right)^{-1}},
\end{aligned}
$$


$\mathbf{D}_{A}$ and $\operatorname{cov}_{A}$ denoting, respectively, variance and covariance under $\mathbf{P}_{A}$ and the constant $C_{M}$ (depending on $\alpha, \widetilde{\sigma}_{f}^{2}$, and $\widetilde{\sigma}^{2}$ only) being of order $O\left(\|M\|^{2}\right)$ as $\|M\| \rightarrow \infty$. Furthermore, we have, for any $n \geqslant \alpha$,

$$
\begin{aligned}
\mathbf{P}_{\nu}\left(\sum_{j=1}^{l_{n}-1} S_{j}(f) \geqslant x\right) \leqslant & C_{M} \exp \left\{-\frac{x^{2} \widetilde{\sigma} / \widetilde{\sigma}_{f}}{2\left(n(1+|\widetilde{\rho}|) \widetilde{\sigma} \widetilde{\sigma}_{f}+x M \sqrt{2} / 3\right)}\right\} \\
& +(n-1) \mathbf{P}_{A}\left(\left|S_{A}(f)\right| \geqslant M_{1}\right)+(n-1) \mathbf{P}_{A}\left(\tau_{A} \geqslant M_{2}\right) .
\end{aligned}
$$

Before developping the argument based on the regenerative method, a few remarks are in order at this point.

$\mathrm{R}$ e $\mathrm{m}$ a $\mathrm{rk} 1$ (on block-moment conditions). Notice that conditions (i) and (ii) do not depend on the atom $A$ chosen. Besides, when dealing with the nonregenerative case, these conditions have to be satisfied by a Nummelin (regenerative) extension of the chain $X$ constructed from a small set $S$, namely an accessible set to which $X$ returns in a given number of steps with probability uniformly bounded by below (see [19] for an account on the Nummelin splitting technique). A sufficient condition for the latter to hold is classically that both $\sup _{x \in S} \mathbf{E}_{x}\left[\tau_{S}^{2}\right]$ and $\sup _{x \in S} \mathbf{E}_{x}\left[\left(\sum_{i=1}^{\tau_{S}} f\left(X_{i}\right)\right)^{2}\right]$ are finite. Recall also that these «block» conditions may be replaced by Foster-Lyapunov drift conditions that generally appear as more tractable in practice (refer, e.g., to [15, Chap. 11] for further details).

$\mathrm{R}$ e $\mathrm{m}$ a r k 2 (on constants involved in the exponential term). It is noteworthy that, although the asymptotic distribution of $n^{-1 / 2} \sum_{j=1}^{l_{n}-1} S_{j}(f)$ as $n \rightarrow \infty$ does not depend on the atom $A$ chosen, its distribution for fixed $n$ does (when $f \equiv 1$ for instance, $\sum_{j=1}^{l_{n}-1} S_{j}(f)=\tau_{A}\left(l_{n}\right)-\tau_{A}$ is the time between the first and last visits to the set $A$ before time $n)$. Hence, the constants involved in bound (5) depend on the choice of the atom and ideally the latter should be picked so as to minimize the bound on the right-hand side of (5).

We also point out that in the case where the $X_{i}$ 's are i.i.d. and $f$ is bounded, by taking $A=E$ (so that $\tau_{A} \equiv 1$ ), $M_{1}>\sup _{x \in E}|f(x)|$ and $M_{2}>1$, the term on the right-hand side of (5) (respectively, of (6)) boils down to a Bennett's type bound (respectively, to a Bernstein's type bound; see [1] and [2]).

P r o o f. Treading in the steps of [3] (see also [4] and [13]), the proof is made in four stages as announced in Subsection 2.1: 1) truncating the r.v.'s as in [8], 2) partitioning the probability space according to all fashions for $X$ to regenerate up to time $n, 3$ ) establishing an accurate exponential bound for sums of bounded i.i.d. 1-lattice random vectors, and 4) summing the bounds previously obtained over all subsets of the partition.

Step 1: truncation. By the same kind of truncation trick as in [8], we shall show that the tail of $\sum_{j=1}^{l_{n}-1} S_{j}(f)$ can be bounded by an exponentially decreasing term plus a term related to the tail behavior of the $\left(S_{j}(f), s_{j}\right)$ 's. Let $M_{1}, M_{2}$ be positive thresholds and consider the truncated r.v.'s

$$
\widetilde{S}_{j}(f)=S_{j}(f) \mathbf{I}_{\left\{\left|S_{j}(f)\right| \leqslant M_{1}\right\}} \quad \text { and } \quad \tilde{s}_{j}=s_{j} \mathbf{I}_{\left\{\left|s_{j}\right| \leqslant M_{2}\right\}}, \quad \text { for } j \geqslant 1 .
$$

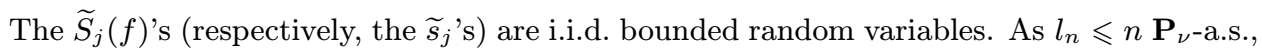
it follows from the union bound that for all positive $x$

$$
\begin{aligned}
\mathbf{P}_{\nu}\left(\sum_{j=1}^{l_{n}-1} S_{j}(f) \geqslant x\right) \leqslant & \mathbf{P}_{\nu}\left(\left\{\sum_{j=1}^{l_{n}-1} \widetilde{S}_{j}(f) \geqslant x\right\} \cap\left\{s_{j}=\widetilde{s}_{j}, 1 \leqslant j \leqslant l_{n}-1\right\}\right) \\
& +(n-1) \mathbf{P}_{A}\left(\left\{\left|S_{A}(f)\right|>M_{1}\right\} \cup\left\{\tau_{A}>M_{2}\right\}\right) .
\end{aligned}
$$

Again the last term on the right-hand side of (7) may be bounded from assumptions on the speed of return time to $A$ and on the tail of $S_{A}(f)$ under $\mathbf{P}_{A}$. We shall now deal with the first term which we denote by $P_{\nu, n}(x)$. 
Step 2: partitioning. In order to be brought to the independent framework, we partition the event $\left\{l_{n} \geqslant 2\right\}$ according to all possible values for the first and last regeneration times, as well as for the total number of regeneration times up to time $n$. In this respect, consider the collection of events

$$
U_{r, l, m}=\left\{\tau_{A}=r, \sum_{j=1}^{m} s_{j}=n-r-l, s_{m+1}>l\right\}
$$

for $1 \leqslant r, l \leqslant n$ and $2 \leqslant m \leqslant n$. Combining the formula of total probability to the strong Markov property, we get

$$
P_{\nu, n}(x)=\sum_{m=2}^{n} \sum_{r=1}^{n} \sum_{l=1}^{n} \mathbf{P}_{\nu}\left(\tau_{A}=r\right) p_{r, l, m}(x) \mathbf{P}_{A}\left(\tau_{A}>l\right),
$$

where $p_{r, l, m}(x)=\mathbf{P}\left(\sum_{1 \leqslant j \leqslant m} \widetilde{S}_{j}(f) \geqslant x, \sum_{1 \leqslant j \leqslant m}\left\{\widetilde{s}_{j}-\mathbf{E}\left[\widetilde{s}_{j}\right]\right\}=n-r-l-m \widetilde{\alpha}\right)$ and $\widetilde{\alpha}=\mathbf{E}\left[\widetilde{s}_{j}\right]$. Since $\mathbf{E}\left[\widetilde{S}_{j}(f)\right] \leqslant 0$, we have $p_{r, l, m}(x) \leqslant \widetilde{p}_{r, l, m}(x)$ for all $r, l$, and $m$, where

$\widetilde{p}_{r, l, m}(x)=\mathbf{P}\left(\frac{1}{\widetilde{\sigma}_{f} \sqrt{m}} \sum_{j=1}^{m}\left\{\widetilde{S}_{j}(f)-\mathbf{E}\left[\widetilde{S}_{j}(f)\right]\right\} \geqslant \frac{x}{\widetilde{\sigma}_{f} \sqrt{m}}, \frac{1}{\widetilde{\sigma}_{A} \sqrt{m}} \sum_{j=1}^{m}\left\{\widetilde{s}_{j}-\mathbf{E}\left[\widetilde{s}_{j}\right]\right\}=\lambda_{r, l, m}\right)$,

with $\lambda_{r, l, m}=(n-r-l-\widetilde{\alpha} m) /\left(\widetilde{\sigma}_{A} \sqrt{m}\right)$. Hence all boils down to get an accurate bound for $p_{r, l, m}(x)$ using the fact that $\left(\widetilde{S}_{j}(f), \widetilde{s}_{j}\right)_{j \geqslant 1}$ is a sequence of bounded i.i.d. bivariate 1-lattice random vectors. With the notation previously set out, notice that $\widetilde{\rho}=\left(\widetilde{\sigma}_{f} \widetilde{\sigma}_{A}\right)^{-1} \operatorname{cov}\left(\widetilde{S}_{j}(f), \widetilde{s}_{j}\right)$ for $j \geqslant 1$.

Step 3: exponential bound for 1-lattice random vectors. It is easy to get a crude exponential estimate for (9) by the standard Chernov method (for instance, see [20] and inequality (22)). However such an estimate would not be precise enough to get a summable bound in $m$ for (8) (preventing thus from reproducing the argument in [13] or [3] for instance). Indeed, under adequate assumptions, from limit theorems for $k$-lattice random vectors with minimum span $h$ (see [6], [7] or Lemma 6.4 in [3]), $\widetilde{p}_{r, l, m}(x)$ can be shown as asymptotically equivalent to $m^{-1 / 2} h \int_{x}^{\infty} \varphi_{\Sigma}\left(t / \sqrt{m}, \lambda_{r, l, m}\right) d t$, where $\varphi_{\Sigma}$ denotes the density of the bivariate Gaussian distribution with mean zero and covariance matrix $\Sigma=$ $\mathbf{D}\left(\widetilde{\sigma}_{f}^{-1} \widetilde{S}_{j}(f), \widetilde{\sigma}_{A}^{-1} \widetilde{s}_{j}\right)$. As pointed out in $[3, \S 6.2 .2]$, the factor $m^{-1 / 2}$ appearing in the latter quantity is of prime importance for obtaining bounds that are summable in $m$. In order to prove the exponential inequality required, we use the method proposed in [24] for improving exponential bounds in the independent setting, which is based on a refined use of the classical argument of the Bahadur-Rao theorem. An exponential bound for sums of i.i.d. 1-lattice bounded random vectors is stated in the following lemma, whose proof is given in Section 3.

Lemma 1 (exponential inequalities for 1-lattice bounded random vectors). Let $\left(S_{j}^{*}, L_{j}^{*}\right)_{1 \leqslant j \leqslant m}$ be i.i.d. centered and square-integrable bivariate r.v.'s with covariance matrix $\Sigma=\left(\begin{array}{ll}\rho & 1 \\ 1\end{array}\right)$. Assume further that the $L_{j}^{*}$ 's are lattice r.v.'s with minimal span $h>0$ and that there exist finite constants $B_{1}$ and $B_{2}$ such that $\left|S_{j}^{*}\right| \leqslant B_{1}$ and $\left|L_{j}^{*}\right| \leqslant B_{2}$ for $j=1, \ldots, m$. Set $B^{2}=B_{1}^{2}+B_{2}^{2}$, then there exists a universal constant $c$ such that for all $m \geqslant 1$ and $y \geqslant 0$,

$$
\mathbf{P}\left(m^{-1 / 2} \sum_{j=1}^{m} S_{j}^{*} \geqslant y, m^{-1 / 2} \sum_{j=1}^{m} L_{j}^{*}=\lambda\right) \leqslant e^{3 / 2}\left(\frac{h}{2 \pi \sqrt{m}}+4 c \frac{B}{m}\right) \exp \left\{-\frac{y^{2}+\lambda^{2}}{4 B^{2}}\right\} .
$$

Furthermore, we also have the following Bennett-Bernstein type inequalities:

$$
\mathbf{P}\left(m^{-1 / 2} \sum_{j=1}^{m} S_{j}^{*} \geqslant y, m^{-12} \sum_{j=1}^{m} L_{j}^{*}=\lambda\right)
$$




$$
\begin{aligned}
& \leqslant e^{3 / 2}\left(\frac{h}{2 \pi \sqrt{m}}+\frac{4 c B}{m}\right) \exp \left\{-\frac{m(1+|\rho|)}{B^{2}} H\left(\frac{B\|(y, \lambda)\|}{(1+|\rho|) \sqrt{m}}\right)\right\} \\
& \leqslant e^{3 / 2}\left(\frac{h}{2 \pi \sqrt{m}}+\frac{4 c B}{m}\right) \exp \left\{-\frac{\|(y, \lambda)\|^{2}}{2(1+|\rho|+B\|(y, \lambda)\| /(3 \sqrt{m}))}\right\} .
\end{aligned}
$$

$\mathrm{R}$ e $\mathrm{m}$ a $\mathrm{r} \mathrm{k} 3$. An overestimated value of the constant $c$ may be deduced by a careful examination of the proof of limit theorems stated in [6] and [7] (see Section 3.1 further). However we do not know at present what the best value for the constant $c$ might be.

Going back to our problem, notice first that, as the $L_{j}^{*}$ 's are lattice with minimal span $h=\widetilde{\sigma}_{A}^{-1}$, Lemma 1 applies to the sequence $\left\{\left(\widetilde{S}_{j}(f)-\mathbf{E}\left[\widetilde{S}_{j}(f)\right]\right) / \widetilde{\sigma}_{f},\left(\widetilde{s}_{j}-\mathbf{E}\left[\widetilde{s}_{j}\right]\right) / \widetilde{\sigma}_{A}\right\}_{j \geqslant 1}$ with $\rho=\widetilde{\rho}, B_{1}=2 M_{1} / \widetilde{\sigma}_{f}$ and $B_{2}=2 M_{2} / \widetilde{\sigma}_{A}$. Observing that $B=\left(B_{1}^{2}+B_{2}^{2}\right)^{1 / 2} \leqslant$ $2\|M\| / \widetilde{\sigma}$, this yields

$$
\begin{aligned}
\widetilde{p}_{r, l, m}(x) \leqslant & e^{3 / 2}\left(\frac{1}{2 \pi \widetilde{\sigma}_{A} \sqrt{m}}+8 c \frac{\|M\|}{\widetilde{\sigma} m}\right) \\
& \times \exp \left\{-\frac{m(1+|\widetilde{\rho}|) \widetilde{\sigma}^{2}}{\|M\|^{2}} H\left(\frac{\|M\| \cdot\left\|\left(x /\left(\widetilde{\sigma}_{f} \sqrt{m}\right), \lambda_{r, l, m}\right)\right\|}{(1+|\widetilde{\rho}|) \widetilde{\sigma} \sqrt{m}}\right)\right\} .
\end{aligned}
$$

Recall that, for any $a>0$, the function $x \mapsto H(a \sqrt{x})$ is concave on $\mathbf{R}^{+}$. Writing

$$
\left\|\left(\frac{x}{\widetilde{\sigma}_{f} \sqrt{m}}, \lambda_{r, l, m}\right)\right\|=\left(\frac{1}{2}\left(\frac{x \sqrt{2}}{\widetilde{\sigma}_{f} \sqrt{m}}\right)^{2}+\frac{1}{2}\left(\lambda_{r, l, m} \sqrt{2}\right)^{2}\right)^{1 / 2},
$$

by concavity arguments we get that

$$
\begin{aligned}
\widetilde{p}_{r, l, m}(x) \leqslant & e^{3 / 2}\left(\frac{1}{2 \pi \widetilde{\sigma}_{A} \sqrt{m}}+8 c \frac{\|M\|}{\widetilde{\sigma} m}\right) \exp \left\{-\frac{m(1+|\widetilde{\rho}|) \widetilde{\sigma}^{2}}{2\|M\|^{2}}\right. \\
& \left.\times\left\{H\left(\frac{\|M\| x \sqrt{2}}{(1+|\widetilde{\rho}|) \widetilde{\sigma}_{f} \widetilde{\sigma} m}\right)+H\left(\frac{\|M\| \cdot\left|\lambda_{r, l, m}\right| \sqrt{2}}{(1+|\widetilde{\rho}|) \widetilde{\sigma} \sqrt{m}}\right)\right\}\right\} .
\end{aligned}
$$

As the function $x \mapsto H(x) / x$ is increasing on $\mathbf{R}^{+}$, we have for any $m \leqslant n$

$$
\begin{aligned}
\exp \{- & \left.\frac{m(1+|\widetilde{\rho}|) \widetilde{\sigma}^{2}}{2\|M\|^{2}} H\left(\frac{\|M\| x \sqrt{2}}{(1+|\widetilde{\rho}|) \widetilde{\sigma}_{f} \widetilde{\sigma} m}\right)\right\} \\
& \leqslant \exp \left\{-\frac{n(1+|\widetilde{\rho}|) \widetilde{\sigma}^{2}}{2\|M\|^{2}} H\left(\frac{\|M\| x \sqrt{2}}{(1+|\widetilde{\rho}|) \widetilde{\sigma}_{f} \widetilde{\sigma} n}\right)\right\} \\
& \leqslant \exp \left\{-\frac{x^{2} \widetilde{\sigma} / \widetilde{\sigma}_{f}}{2\left(n(1+|\widetilde{\rho}|) \widetilde{\sigma} \widetilde{\sigma}_{f}+x\|M\| \sqrt{2} / 3\right)}\right\},
\end{aligned}
$$

the last bound following from the classical inequality $H(x) \geqslant x^{2} /(2(1+x / 3)), x \geqslant 0$.

Step 4: control of the sum. Now combining the previous bound to (8) and (12), we deduce that

$$
P_{n, \nu}(x) \leqslant e^{3 / 2} \exp \left\{-\frac{n(1+|\widetilde{\rho}|) \widetilde{\sigma}^{2}}{2\|M\|^{2}} H\left(\frac{\|M\| x \sqrt{2}}{(1+|\widetilde{\rho}|) \widetilde{\sigma}_{f} \widetilde{\sigma} n}\right)\right\} \Gamma_{n}
$$

where

$$
\Gamma_{n}=\sum_{m=2}^{n} \sum_{r=1}^{n} \sum_{l=1}^{n} \mathbf{P}_{\nu}\left(\tau_{A}=r\right) \mathbf{P}_{A}\left(\tau_{A}>l\right)\left(\frac{c_{1}}{\sqrt{m}}+\frac{c_{2}}{m}\right) \gamma_{r, l, m},
$$

with $c_{1}=\left(2 \pi \widetilde{\sigma}_{A}\right)^{-1}, c_{2}=8 c\|M\| / \widetilde{\sigma}$, and

$$
\gamma_{r, l, m}=\exp \left\{-\frac{m(1+|\widetilde{\rho}|) \widetilde{\sigma}^{2}}{2\|M\|^{2}} H\left(\frac{\|M\| \cdot\left|\lambda_{r, l, m}\right| \sqrt{2}}{(1+|\widetilde{\rho}|) \widetilde{\sigma} \sqrt{m}}\right)\right\}
$$




$$
\leqslant \exp \left\{-\frac{\lambda_{r, l, m}^{2}}{2\left(1+|\widetilde{\rho}|+\left|\lambda_{r, l, m}\right|\|M\| \sqrt{2} /(3 \widetilde{\sigma} \sqrt{m})\right.}\right\} .
$$

Recall that $\lambda_{r, l, m}=(n-r-l-\widetilde{\alpha} m) /\left(\widetilde{\sigma}_{A} \sqrt{m}\right)$ and consider the subdivision defined by the points

$$
a_{n, m}=\frac{n-\widetilde{\alpha} m}{\widetilde{\sigma}_{A} \sqrt{m}}, \quad \text { for } 1 \leqslant m \leqslant n .
$$

In order to bound this sum, we split the latter into two parts, according to whether $r+l$ is less than $\widetilde{\sigma}_{A} \sqrt{m}$ or not. Since $\gamma_{r, l, m} \leqslant 1$, by repeated use of the Markov inequality, we get

$$
\begin{aligned}
& \sum_{m=2}^{n} \sum_{r+l>\tilde{\sigma}_{A} \sqrt{m}}^{n} \mathbf{P}_{\nu}\left(\tau_{A}=r\right) \mathbf{P}_{A}\left(\tau_{A}>l\right)\left(\frac{c_{1}}{\sqrt{m}}+\frac{c_{2}}{m}\right) \gamma_{r, l, m} \\
& \leqslant \sum_{m=1}^{n}\left(\frac{c_{1}}{\sqrt{m}}+\frac{c_{2}}{m}\right) \sum_{l \geqslant \tilde{\sigma}_{A} \sqrt{m}}^{n} \mathbf{P}_{A}\left(\tau_{A}>l\right) \\
& \leqslant \frac{\mathbf{E}_{A}\left[\tau_{A}^{2}\right]}{\widetilde{\sigma}_{A}^{2}} \sum_{m=1}^{n}\left(\frac{c_{1}}{m^{3 / 2}}+\frac{c_{2}}{m^{2}}\right) \leqslant \frac{\mathbf{E}_{A}\left[\tau_{A}^{2}\right]}{\widetilde{\sigma}_{A}^{2}}\left(3 c_{1}+c_{2} \frac{\pi^{2}}{6}\right)
\end{aligned}
$$

From the inequality $(a-b)^{2} \geqslant a^{2} / 2-b^{2}$, it follows that $\lambda_{r, l, m}^{2} \geqslant a_{n, m}^{2} / 2-\left((r+l) /\left(\widetilde{\sigma}_{A} \sqrt{m}\right)\right)^{2}$ and, for $r+l \leqslant \widetilde{\sigma}_{A} \sqrt{m}$,

$$
\lambda_{r, l, m}^{2} \geqslant\left(a_{n, m}^{2} / 2-1\right)_{+},
$$

where $a_{+}$denotes the positive part of any real number $a$. The term on the right-hand side of (15) being decreasing in $\lambda_{r, l, m}$, we have

$$
\gamma_{r, l, m} \leqslant \exp \left\{-\frac{\left(a_{n, m}^{2} / 2-1\right)_{+}}{2\left(1+|\widetilde{\rho}|+\left(a_{n, m}^{2} / 2-1\right)_{+}^{1 / 2}\|M\| \sqrt{2} /(3 \widetilde{\sigma} \sqrt{m})\right)}\right\} .
$$

Consider the set $A_{n}=\left\{m \in\{2, \ldots, n\}:\left(a_{n, m}^{2} / 2-1\right)_{+}^{1 / 2}\|M\| \sqrt{2} /(3 \widetilde{\sigma}) \leqslant m^{1 / 2}(1+|\widetilde{\rho}|)\right\}$ of indexes $m$ for which the «Bernstein type» bound (17) describes a Gaussian tail behavior (the complement $A_{n}^{c}$ corresponding to indexes $m$ for which (17) provides a Poisson type estimate). On the one hand, if $m \in A_{n}$, then

$$
\gamma_{r, l, m} \leqslant \exp \left\{-\frac{a_{n, m}^{2} / 2-1}{4(1+|\widetilde{\rho}|)}\right\}
$$

and on the other hand, if $m \in A_{n}^{c}$, then

$$
\gamma_{r, l, m} \leqslant \exp \left\{-\frac{3 \widetilde{\sigma} \sqrt{m}\left(a_{n, m}^{2} / 2-1\right)_{+}^{1 / 2}}{4\|M\| \sqrt{2}}\right\} \leqslant \exp \left\{-\frac{9 \widetilde{\sigma}^{2}(1+|\widetilde{\rho}|)}{8\|M\|^{2}} m\right\} .
$$

It follows that

$$
\sum_{m=1}^{n} \sum_{r+l \leqslant \tilde{\sigma}_{A} \sqrt{m}}^{n} \mathbf{P}_{\nu}\left(\tau_{A}=r\right) \mathbf{P}_{A}\left(\tau_{A}>l\right)\left(\frac{c_{1}}{\sqrt{m}}+\frac{c_{2}}{m}\right) \gamma_{r, l, m} \leqslant \alpha\left(c_{1}+c_{2}\right)\left(U_{n}+V_{n}\right),
$$

where $U_{n}=\sum_{m \in A_{n}} m^{-1 / 2} \gamma_{r, l, m}$ and $V_{n}=\sum_{m \in A_{n}^{c}} m^{-1 / 2} \gamma_{r, l, m}$. As in Step 4 of Theorem 5.1's proof in [3], we shall prove that $U_{n}$ is bounded by a Riemann sum. Notice that for all $m \leqslant n$, we have $a_{n, m}-a_{n, m+1}=\widetilde{\alpha} \widetilde{\sigma}_{A}^{-1} m^{-1 / 2}+a_{n, m+1}\left(\left(1+m^{-1}\right)^{1 / 2}-1\right)$ and $a_{n, m} \geqslant a_{n, m+1}$. If $a_{n, m+1} \geqslant 0$, then $\widetilde{\alpha} \widetilde{\sigma}_{A}^{-1} m^{-1 / 2} \leqslant a_{n, m}-a_{n, m+1}$. On the other hand, if $a_{n, m+1} \leqslant 0$, we have $-a_{n, m+1}=(\widetilde{\alpha}(m+1)-n) \widetilde{\sigma}_{A}^{-1} m^{-1 / 2} \leqslant \widetilde{\alpha} \widetilde{\sigma}_{A}^{-1} m^{1 / 2}$ provided that $n \geqslant \widetilde{\alpha}$. Hence, for any $m$ such that $a_{n, m+1} \leqslant 0$,

$$
\widetilde{\alpha} \widetilde{\sigma}_{A}^{-1} m^{-1 / 2} \leqslant a_{n, m}-a_{n, m+1}+2^{-1} \widetilde{\alpha} \widetilde{\sigma}_{A}^{-1} m^{-1 / 2},
$$

and consequently for any $m \geqslant 2$ and $n \geqslant \widetilde{\alpha}$, we have

$$
\widetilde{\alpha} \widetilde{\sigma}_{A}^{-1} m^{-1 / 2} \leqslant 2\left(a_{n, m}-a_{n, m+1}\right) .
$$


We thus obtain that

$$
\begin{aligned}
U_{n} & \leqslant 2 \widetilde{\alpha}^{-1} \widetilde{\sigma}_{A} e^{c_{3}} \sum_{m=1}^{n}\left(a_{n, m}-a_{n, m+1}\right) \exp \left\{-\frac{c_{3} a_{n, m}^{2}}{2}\right\} \\
& \leqslant 2 \widetilde{\alpha}^{-1} \widetilde{\sigma}_{A} e^{c_{3}} \int_{-\infty}^{\infty} \exp \left\{-\frac{c_{3} x^{2}}{2}\right\} d x=2 \widetilde{\alpha}^{-1} \widetilde{\sigma}_{A} \sqrt{\frac{2 \pi}{c_{3}}} e^{c_{3}},
\end{aligned}
$$

with $c_{3}=1 /(4(1+|\widetilde{\rho}|)) \in(1 / 8,1 / 4)$ (in particular, $\left.e^{c_{3}} / \sqrt{c_{3}} \leqslant 2 \sqrt{2} e^{1 / 4}\right)$. Besides, proceeding in a similar way, we get

$$
\begin{aligned}
V_{n} & \leqslant \sum_{m \in A_{n}^{c}} m^{-1 / 2} \exp \left\{-\frac{9(1+|\widetilde{\rho}|) \widetilde{\sigma}^{2}}{8\|M\|^{2}} m\right\} \leqslant \int_{0}^{\infty} x^{-1 / 2} \exp \left\{-\frac{9(1+|\widetilde{\rho}|) \widetilde{\sigma}^{2}}{8\|M\|^{2}} x\right\} d x \\
& =\frac{2 \sqrt{2}\|M\| \sqrt{\pi}}{3 \widetilde{\sigma}(1+|\widetilde{\rho}|)^{1 / 2}} \leqslant \frac{2 \sqrt{2}\|M\| \sqrt{\pi}}{3 \widetilde{\sigma}}
\end{aligned}
$$

Now combining (16), (18), and (19), we obtain $\Gamma_{n} \leqslant c_{4}$ with

$$
c_{4}=\frac{\sigma_{A}^{2}+\alpha^{2}}{\widetilde{\sigma}_{A}^{2}}\left(3 c_{1}+c_{2} \frac{\pi^{2}}{6}\right)+2 \widetilde{\sigma}_{A}\left(c_{1}+c_{2}\right) e^{c_{3}} \frac{\alpha}{\widetilde{\alpha}} \sqrt{\frac{2 \pi}{c_{3}}}+\left(c_{1}+c_{2}\right) \frac{2 \sqrt{2}\|M\| \alpha \sqrt{\pi}}{3 \widetilde{\sigma}} .
$$

Using (14), this yields the tail bound (5), where $C_{M}=e^{3 / 2} c_{4}$ with $c_{4}$ given by (20). Notice that $C_{M}=O\left(\|M\|^{2}\right)$ as $\|M\| \rightarrow \infty$. And using (13) we obtain the tail bound (6) with the «Bernstein version» for the exponential term.

\section{Technical details — proof of Lemma 1. Set}

$$
\mathscr{S}_{m}=m^{-1 / 2} \sum_{i \leqslant n} S_{j}^{*} \quad \text { and } \quad \mathscr{L}_{m}=m^{-1 / 2} \sum_{i \leqslant n} L_{j}^{*}
$$

for notational convenience. For $u=\left(u_{1}, u_{2}\right) \in \mathbf{R}^{+} \times \mathbf{R}$, let $\psi_{m}(u)=$ $\ln \mathbf{E}\left[\exp \left\{\left\langle u,\left(\mathscr{S}_{m}, \mathscr{L}_{m}\right)\right\rangle\right\}\right]$ be the log-Laplace of the random vector $\left(\mathscr{S}_{m}, \mathscr{L}_{m}\right)$ and denote by $\psi_{u, m}^{(1)}$ and $\psi_{u, m}^{(2)}$ its gradient and its Hessian matrix. Consider now the probability measure $\mathbf{P}_{u, m}$ defined by the Esscher transformation $d \mathbf{P}_{u, m}=\exp \left\{\left\langle u,\left(\mathscr{S}_{m}, \mathscr{L}_{m}\right)\right\rangle-\right.$ $\left.\psi_{m}(u)\right\} d \mathbf{P}$. Expectation under $\mathbf{P}_{u, m}$ is denoted by $\mathbf{E}_{u, m}[\cdot]$ in what follows. By exponential change of probability measure, we get

$$
\begin{aligned}
\mathbf{P}\left(\mathscr{S}_{m} \geqslant y, \mathscr{L}_{m}=\lambda\right) & =\mathbf{E}_{u, m}\left[e^{\psi_{m}(u)-\left\langle u,\left(\mathscr{S}_{m}, \mathscr{L}_{m}\right)\right\rangle} \mathbf{I}_{\left\{\mathscr{S}_{m} \geqslant y, \mathscr{L}_{m}=\lambda\right\}}\right] \\
& =e^{-\langle u,(y, \lambda)\rangle-\psi_{m}(u)} \mathbf{E}_{u, m}\left[e^{-\left\langle u,\left(\mathscr{S}_{m}-y, \mathscr{L}_{m}-\lambda\right)\right\rangle} \mathbf{I}_{\left\{\mathscr{S}_{m} \geqslant y, \mathscr{L}_{m}=\lambda\right\}}\right]
\end{aligned}
$$

Now choose $u=u^{*}$ such that $\psi_{m}^{(1)}\left(u^{*}\right)=(y, \lambda)$, that is,

$$
u^{*}=\arg \sup _{u \in \mathbf{R}_{+} \times \mathbf{R}}\left\{\langle u,(y, \lambda)\rangle-\psi_{m}(u)\right\} .
$$

Since $\mathbf{E}\left[e^{\left\langle u,\left(\mathscr{S}_{m}, \mathscr{L}_{m}\right)\right\rangle}\right]=e^{\psi_{m}(u)}$, by differentiating one obtains

$$
\mathbf{E}\left[e^{\left\langle u^{*},\left(\mathscr{S}_{m}, \mathscr{L}_{m}\right)\right\rangle}\left(\mathscr{S}_{m}, \mathscr{L}_{m}\right)\right]=\psi_{m}^{(1)}\left(u^{*}\right) e^{\psi_{m}\left(u^{*}\right)}=(y, \lambda) e^{\psi_{m}\left(u^{*}\right)}
$$

yielding $\mathbf{E}_{u^{*}, m}\left[\left(\mathscr{S}_{m}, \mathscr{L}_{m}\right)\right]=(y, \lambda)$ and $\mathbf{D}_{u^{*}, m}\left[\left(\mathscr{S}_{m}, \mathscr{L}_{m}\right)\right]=\psi_{m}^{(2)}\left(u^{*}\right)$ in a similar fashion, denoting by $\mathbf{D}_{u^{*}, m}[\cdot]$, the variance-covariance matrix under $\mathbf{P}_{u^{*}, m}$. By integrating by parts combined with straightforward changes of variables, one obtains

$$
\begin{aligned}
A_{m}(u) & =\mathbf{E}_{u, m}\left[e^{-\left\langle u,\left(\mathscr{S}_{m}-y, \mathscr{L}_{m}-\lambda\right)\right\rangle} \mathbf{I}_{\left\{\mathscr{S}_{m} \geqslant y, \mathscr{L}_{m}=\lambda\right\}}\right] \\
& =\int e^{-\left\langle u,\left(\mathscr{S}_{m}-y, \mathscr{L}_{m}-\lambda\right)\right\rangle} \mathbf{I}_{\left\{\mathscr{S}_{m}-y \geqslant 0, \mathscr{L}_{m}-\lambda=0\right\}} d \mathbf{P}_{u, m}
\end{aligned}
$$




$$
\begin{aligned}
= & \mathbf{P}_{u, m}\left(S_{m} \geqslant y, L_{m}=\lambda\right) \\
& -u_{1} \int_{y}^{\infty} e^{-u_{1}(s-y)} \mathbf{P}_{u, m}\left(S_{m}-y \geqslant s-y, L_{m}-\lambda=0\right) d s \\
\leqslant & u_{1} \int_{0}^{\infty} e^{-u_{1} s} \mathbf{P}_{u, m}\left(S_{m}-y \geqslant 0, L_{m}-\lambda=0\right) \\
& -\mathbf{P}_{u, m}\left(S_{m}-y \geqslant s, L_{m}-\lambda=0\right) d s .
\end{aligned}
$$

Using the results in [6] and [7] or the local Berry-Esseen bound proved in [4] (see Theorem 4), we know that there exists a constant $C_{u}$ such that, uniformly in $(y, \lambda)$,

$$
\left|\mathbf{P}_{u, m}\left(\mathscr{S}_{m}-y \geqslant s, \mathscr{L}_{m}-\lambda=0\right)-\frac{h}{\sqrt{m}} \int_{s}^{\infty} \varphi_{W_{u}}(t, 0) d t\right| \leqslant C_{u} m^{-1},
$$

where $W_{u}=\mathbf{D}_{u, m}\left[\left(\mathscr{S}_{m}, \mathscr{L}_{m}\right)\right]$ and $\varphi_{W_{u}}$ is the density of the bivariate Gaussian distribution with covariance matrix $W_{u}$. From [6] (see also [7]) a crude bound for $C_{u}$ is given by $c k_{3}\left(\mathbf{P}_{u, 1}\right)$, where $k_{3}\left(\mathbf{P}_{u, 1}\right)=\mathbf{E}_{u, 1}\left[\left|X-\mathbf{E}_{u, 1}[X]\right|^{3}\right] /\left(\mathbf{E}_{u, 1}\left[\left(X-\mathbf{E}_{u, m}[X]\right)^{2}\right]\right)^{3 / 2}$ and $c$ is a universal constant. Notice that

$$
\varphi_{W_{u}}(s, 0)=\frac{\exp \left\{-s^{2} /\left(2 \alpha_{u, m}^{2}\right)\right\}}{\sqrt{2 \pi \mathbf{D}_{u, m}\left(\mathscr{L}_{m}\right)} \sqrt{2 \pi \alpha_{u, m}^{2}}},
$$

with

$$
\alpha_{u, m}=\left(\mathbf{D}_{u, m}\left(\mathscr{S}_{m}\right)\right)^{1 / 2}\left(1-\rho_{u, m}^{2}\right)^{1 / 2}, \quad \rho_{u, m}^{2}=\frac{\left(\operatorname{cov}_{u, m}\left(\mathscr{S}_{m}, \mathscr{L}_{m}\right)\right)^{2}}{\mathbf{D}_{u, m}\left(\mathscr{L}_{m}\right) \mathbf{D}_{u, m}\left(\mathscr{S}_{m}\right)} .
$$

This yields for $u=u^{*}$,

$$
\begin{aligned}
A_{m}\left(u^{*}\right) & \leqslant u_{1}^{*} \int_{0}^{\infty} e^{-u_{1}^{*} s}\left(\frac{h}{\sqrt{m}} \int_{0}^{s} \varphi_{W_{u^{*}}}(t, 0) d t+2 C_{u^{*}} m^{-1}\right) d s \\
& =\frac{h}{\sqrt{m}} \int_{0}^{\infty} e^{-u_{1}^{*} s} \varphi_{W_{u^{*}}}(s, 0) d s+2 C_{u^{*}} m^{-1} \\
& =\frac{h}{\sqrt{m}} \frac{1}{\sqrt{2 \pi \mathbf{D}_{u^{*}, m}\left(\mathscr{L}_{m}\right)}} \frac{\bar{\Phi}\left(u_{1}^{*} \alpha_{u, m}\right)}{2 \pi \varphi\left(u_{1}^{*} \alpha_{u, m}\right)}+2 C_{u^{*}} m^{-1},
\end{aligned}
$$

where $\varphi(x)$ denotes the density of the standard normal distribution and $\bar{\Phi}(x)$ its survivor function. Recall that for all $x>0$ (see [24]),

$$
\frac{1}{\sqrt{2 \pi}(1+x)} \leqslant \frac{\bar{\Phi}(x)}{\sqrt{2 \pi} \varphi(x)} \leqslant \frac{1}{\sqrt{2 \pi} \max (x, 1)}
$$

which leads to

$$
A_{m}\left(u^{*}\right) \leqslant \frac{h}{\sqrt{m}} \frac{1}{2 \pi\left(\mathbf{D}_{u^{*}, m}\left(\mathscr{L}_{m}\right)\right)^{1 / 2}}+2 C_{u^{*}} m^{-1} .
$$

The matter is now to find an upper bound for the quantity

$$
L\left(u^{*}\right)=e^{-\left\langle u^{*},(y, \lambda)\right\rangle-\psi_{m}\left(u^{*}\right)}=\inf _{u} e^{-\left(\langle u,(y, \lambda)\rangle-\psi_{m}(u)\right)} .
$$

Since $\left\langle u,\left(\mathscr{S}_{m}, \mathscr{L}_{m}\right)\right\rangle \leqslant 1+\|u\|^{2} B^{2}$, we have $\mathbf{E}\left[e^{\left\langle u,\left(\mathscr{S}_{m}, \mathscr{L}_{m}\right)\right\rangle}\right] \leqslant e \exp \left\{\|u\|^{2} B^{2}\right\}$ for all $u$, so that

$$
L\left(u^{*}\right) \leqslant e \exp \left(-\frac{y^{2}+\lambda^{2}}{4 B^{2}}\right) .
$$

It only remains to bound $k_{3}\left(\mathbf{P}_{u^{*}, m}\right)$ explicitely. Using (22), observe first that

$$
\begin{aligned}
\mathbf{E}_{u^{*}, 1}\left[\left(\mathscr{S}_{1}-\mathbf{E}_{u^{*}, 1}\left[\mathscr{S}_{1}\right]\right)^{2}\right] & =\mathbf{E}_{u^{*}, m}\left[\left(\mathscr{S}_{m}-\mathbf{E}_{u^{*}, m}\left[\mathscr{S}_{m}\right]\right)^{2}\right] \\
& =\int(s-y)^{2} e^{\left\langle u^{*},(s, l)\right\rangle-\psi_{m}\left(u^{*}\right)} d \mathbf{P}(s, l)
\end{aligned}
$$




$$
\begin{aligned}
& \geqslant e^{-1} \int(s-y)^{2} e^{\left(s^{2}+l^{2}\right) /\left(4 B^{2}\right)} d \mathbf{P}(s, l) \\
& \geqslant e^{-1} \int(s-y)^{2} d \mathbf{P}(s, l)=e^{-1}\left(1+y^{2}\right) \geqslant e^{-1},
\end{aligned}
$$

and similarly $\mathbf{D}_{u^{*}, m}\left(\mathscr{L}_{m}\right) \geqslant e^{-1}$. Moreover, since we have the bound

$$
\mathbf{E}_{u^{*}, 1}\left[\left|\mathscr{S}_{1}-\mathbf{E}_{u^{*}, 1}\left[\mathscr{S}_{1}\right]\right|^{3}\right] \leqslant 2 B \mathbf{D}_{u^{*}, 1}\left(\mathscr{S}_{1}\right)
$$

it follows from (23) that

$$
k_{3}\left(\mathbf{P}_{u^{*}, 1}\right) \leqslant 2 e^{1 / 2} B .
$$

The desired result follows by combining (21) with (22) and estimate (25).

To obtain the Bernstein-Bennett type bound, we replace the crude bound (22) by a more refined one by using standard arguments. We have

$$
\mathbf{E}\left[e^{\left\langle u,\left(\mathscr{S}_{m}, \mathscr{L}_{m}\right)\right\rangle}\right]=\prod_{i=1}^{m} \mathbf{E}\left[\exp \left\{\frac{u_{1}}{\sqrt{m}} S_{i}^{*}+\frac{u_{2}}{\sqrt{m}} L_{i}^{*}\right\}\right] .
$$

Since $s \mapsto\left(e^{s}-1-s\right) / s^{2}$ is increasing on $\mathbf{R}_{+}^{*}$ and $\left|u_{1} m^{-1 / 2} S_{i}^{*}+u_{2} m^{-1 / 2} L_{i}^{*}\right| \leqslant m^{-1 / 2}\|u\| B$, we have

$$
\begin{gathered}
\mathbf{E}\left[\exp \left\{\frac{u_{1}}{\sqrt{m}} S_{i}^{*}+\frac{u_{1}}{\sqrt{m}} L_{i}^{*}\right\}\right] \leqslant \mathbf{E}\left[1+\frac{u_{1}}{\sqrt{m}} S_{i}^{*}+\frac{u_{1}}{\sqrt{m}} L_{i}^{*}\right] \\
+\mathbf{E}\left[\left(u_{1} S_{i}^{*}+u_{2} L_{i}^{*}\right)^{2}\right] \frac{\exp \left\{m^{-1 / 2}\|u\| B\right\}-1-m^{-1 / 2}\|u\| B}{\|u\|^{2} B^{2}} \\
\leqslant 1+(1+|\rho|) \frac{\exp \left\{m^{-1 / 2}\|u\| B\right\}-1-m^{-1 / 2}\|u\| B}{B^{2}} .
\end{gathered}
$$

The last inequality follows from the trivial inequality

$$
\mathbf{E}\left[\left(u_{1} S_{i}^{*}+u_{2} L_{i}^{*}\right)^{2}\right]=u_{1}^{2}+2 \rho u_{1} u_{2}+u_{2}^{2} \leqslant u_{1}^{2}+|\rho|\left(u_{1}^{2}+u_{2}^{2}\right)+u_{2}^{2} .
$$

Then, we obtain

$$
\begin{aligned}
\mathbf{E}\left[e^{\left\langle u,\left(\mathscr{S}_{m}, \mathscr{L}_{m}\right)\right\rangle}\right] & \leqslant\left(1+\frac{1+|\rho|}{B^{2}}\left(\exp \left\{m^{-1 / 2}\|u\| B\right\}-1-m^{-1 / 2}\|u\| B\right)\right)^{m} \\
& \leqslant \exp \left\{m \frac{1+|\rho|}{B^{2}}\left(\exp \left\{m^{-1 / 2}\|u\| B\right\}-1-m^{-1 / 2}\|u\| B\right)\right\} .
\end{aligned}
$$

If we choose $u$ such that $\|u\|=m^{1 / 2} B^{-1} \ln \left(1+m^{-1 / 2}(1+|\rho|)^{-1} B\|(y, \lambda)\|\right)$ and $u$ colinear to $(y, \lambda)$, then we get

$$
\begin{aligned}
L\left(u^{*}\right) & \leqslant \exp \left\{-\|u\| \cdot\|(y, \lambda)\|+m \frac{1+|\rho|}{B^{2}}\left(\exp \left\{m^{-1 / 2}\|u\| B\right\}-1-m^{-1 / 2}\|u\| B\right)\right\} \\
& =\exp \left\{-m \frac{1+|\rho|}{B^{2}} H\left(\frac{B\|(y, \lambda)\|}{m^{1 / 2}(1+|\rho|)}\right)\right\},
\end{aligned}
$$

where $H(x)=(1+x) \ln (1+x)-x$. The last inequality follows from the classical inequality $H(x) \geqslant x^{2} /(2(1+x / 3))$, for $x \geqslant 0$.

\section{REFERENCES}

1. Bennett $S$. Probability inequalities for a sum of independent random variables. - J. Amer. Statist. Assoc., 1962, v. 57, p. 33-45.

2. Бернштейн C. H. Об одном видоизменении неравенства Чебышёва и погрешности формулы Лапласа. - Уч. зап. Н.-И. кафедры Украины, 1924; Собрание сочинений, т. 4. М.: Наука, 1964, с. 71-80. 
3. Bertail P., Clémençon $S$. Edgeworth expansions for suitably normalized sample mean statistics of atomic Markov chains. - Probab. Theory Related Fields, 2004, v. 130, p. $388-414$.

4. Bolthausen E. The Berry-Esseen theorem for functionals of discrete Markov chains. Z. Wahrscheinlichkeitstheor. verw. Geb., 1980, v. 54, p. 59-73.

5. Doukhan P. Mixing: Properties and Examples. Berlin: Springer-Verlag, 1994, 82 p. (Lecture Notes in Statist., v. 85.)

6. Дубинскайте Й. Предельные теоремы в $\mathbf{R}^{k}$. I. - Литов. матем. сб., 1982, т. 22, c. $129-140$.

7. Дубинскайте Й. Предельные теоремы в $\mathbf{R}^{k}$. II, III. - Литов. матем. сб., 1984, т. 24 , с. $256-265 ; 325-334$.

8. Фук Д.Х., Нагаев С.В. Вероятностные неравенства для сумм независимых случайных величин. - Теория вероятн. и ее примен., 1971, т. 16 , в. 4, с. 660675 ; исправл.: 1976, т. 21, в. 4.

9. Glynn P.W., Ormoneit D. Hoeffding's inequality for uniformly ergodic Markov chains. - Statist. Probab. Lett., 2002, v. 56, № 2, p. 143-146.

10. Hoeffding $W$. Probability inequalities for sums of bounded random variables. - J. Amer. Statist. Assoc., 1963, v. 58, p. 13-30.

11. Léon C.A., Perron F. Optimal Hoeffding bounds for discrete reversible Markov chains. - Ann. Appl. Probab., 2004, v. 14, № 2, p. 958-970.

12. Lézaud P. Chernoff-type bound for finite Markov chains. - Ann. Appl. Probab., 1998, v. 8, № 3, p. 849-867.

13. Малиновский В. К. О предельных теоремах для харрисовских цепей Маркова. Теория вероятн. и ее примен., 1986, т. 31, в. 2, с. 315-332.

14. Marton K. A measure concentration inequality for contracting Markov chains. Geom. Funct. Anal., 1996, v. 6, № 3, p. 556-571.

15. Meyn S. P., Tweedie R. L. Markov Chains and Stochastic Stability. London: SpringerVerlag London, Ltd., 1993, 548 p.

16. Нагаев C. В. Умножение предельных теорем для однородных цепей Маркова. Теория вероятн. и ее примен., 1961, т. 6 , в. 1, с. $67-86$.

17. Nagaev S. V. Large deviations of sums of independent random variables. - Ann. Probab., 1979 , v. 7, № 5, p. 745-789.

18. Nummelin E. A splitting technique for Harris recurrent chains. - Z. Wahrscheinlichkeitstheor. verw. Geb., 1978, v. 43, p. 309-318.

19. Nummelin E. General Irreducible Markov Chains and Non-negative Operators. Cambridge: Cambridge Univ. Press, 1984, 172 p.

20. Прохоров Ю.В. Распространение неравенств С.Н. Бернштейна на многомерный случай. - Теория вероятн. и ее примен., 1968, т. 13, в. 2, с. 266-274.

21. Rio $E$. Théorie asymptotique des processus aléatoires faiblement dépendants. Berlin: Springer-Verlag, 2000, 169 p.

22. Samson P.-M. Concentration of measure inequalities for Markov chains and $\Phi$-mixing processes. - Ann. Probab., 2000, v. 28, № 1, p. 416-461.

23. Smith W. L. Regenerative stochastic processes. - Proc. Roy. Statist. Soc., Ser. A, 1955 , v. 232 , p. 6-31.

24. Talagrand $M$. The missing factor in Hoeffding's inequalities. - Ann. Inst. H. Poincaré, 1995, v. 31, № 4, p. 689-670.

25. Thorisson H. Coupling, Stationarity, and Regeneration. New York: Springer-Verlag, 2000,517 p.

Поступила в редакцию

24.V.2007

Исправленный вариант 13.XII. 2007 